\title{
IMAGE ENHANCEMENT USING NONSUBSAMPLED CONTOURLET TRANSFORM
}

\author{
Rafia Mumtaz', Raja Iqbal ${ }^{2}$ and Dr.Shoab A.Khan ${ }^{3}$ \\ ${ }^{12}{ }^{2}$ MCS, National Unioversity of Sciences and Technology, Rawalpindi, Pakistan: ${ }^{3}$ EME, \\ National University of Sciences nad Technology, Rawalpindi, Pakistan
}

\begin{abstract}
This paper presents a novel technique of image Enhancement which can be widely used in medical and biological imaging to improve the image quality. The principle objective of enhancement is to process an image so that the result is more suitable than the original image for a specific application. Image enhancement enhances weak edges or weak features in an image while keeping strong edges or features. All existing methods of image enhancement decompose images in a separable fashion, and thus cannot use the geometric information in the transform domain to distinguish weak edges from noises. Therefore, they either amplify noises or introduce visible artifacts, when they are applied to noisy images. The NonSubsampled Contourlet transform built upon NonSubsampled pyramids and NonSubsampled directional filter banks can provide a shift invariant directional multi resolution image representation. The geometric information is gathered pixel by pixel from the NonSubsampled Contourlet Transform coefficients. The proposed method achieved better enhancement results than the wavelet based methods of enhancement.
\end{abstract}

Key words: Enhancement, NonSubsampled, Contourlet Transform.

\section{INTRODUCTION}

The aim of image enhancement is to improve the interpretability or perception of information in images for human viewers, or to provide 'better' input for other automated image processing techniques. It enhances weak edges or weak features in an image while keeping strong edges or features. Traditional image enhancement methods such as unsharp masking, split an image into different frequency subbands 
and amplify the high pass subbands. Although the wavelet transform has been proven to be powerful in many signal and image processing applications such as compression, noise removal, image edge enhancement, and feature extraction; wavelets are not optimal in capturing the two-dimensional singularities found in images. Several transforms have been proposed for image signals that have incorporated directionality and multiresolution and hence, could more efficiently capture edges in natural images [1]. Recently Do and Vetterli proposed an efficient directional multiresolution image representation called the Contourlet transform [2]. The Contourlet transform employs Laplacian pyramids to achieve multiresolution decomposition and directional filter banks to achieve directional de-composition. Due to downsampling and upsampling, the Contourlet transform is shift-variant. However, Shift sensitivity is an undesirable property because it implies that the transform coefficients fail to distinguish between input signal shifts [3]. Shiftinvariance is desirable in image analysis applications such as edge detection, contour characterization, and image enhancement.

In this paper, the Nonsubsampled Contourlet transform (NSCT) is presented, which is a shift-invariant version of the Contourlet transform. The NSCT is built upon iterated nonsubsampled filter banks to obtain a shift-invariant directional multiresolution image representation. Based on the NSCT, a new method for image enhancement is introduced.

\section{CONSTRUCTION}

\subsection{NonSubsampled Contourlet Transform}

The NSCT is a fully shift-invariant, multi-scale, and multidirectional expansion that has a fast implementation. Figure 1 (a) displays the overview of the proposed NSCT. The structure consists in a bank of filters that splits the 2-D frequency plane in the subbands illustrated in Figure 1(b). Our proposed transform can thus be divided into two shift-invariant parts which are as follow:

1. A nonsubsampled pyramid structure that ensures the multi-scale property and

2. A nonsubsampled DFB structure that gives directionality.

The contourlet transform employs Laplacian pyramids for multiscale decomposition, and directional filter banks (DFB) for directional decomposition. To achieve the shift-invariance, the nonsubsampled contourlet transform is built upon nonsubsampled pyramids and nonsubsampled DFB [4]. 


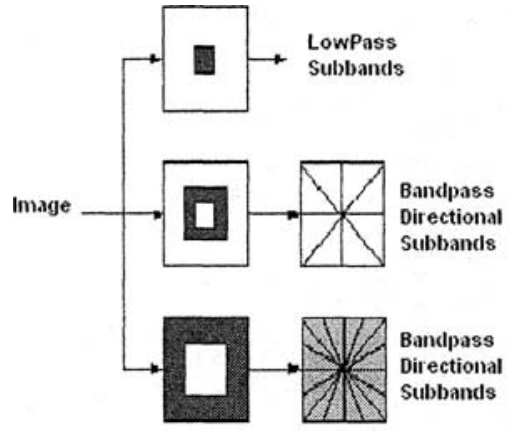

(a)

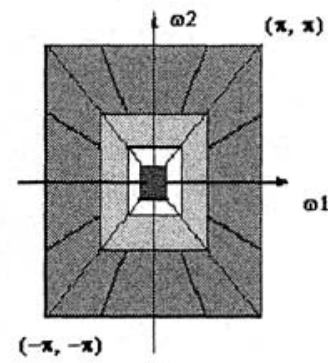

(b)

Figure 1. The NonSubsampled Contourlet Transform. (a) NonSubsampled tilter bank structure that implements the NSCT. (b) The idealized frequency partitioning obtained with the proposed structure

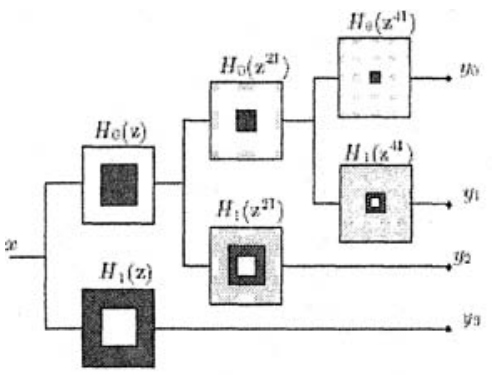

(a)

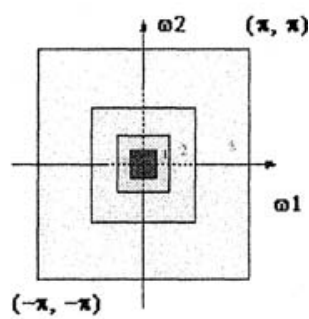

(b)

Figure 2. The proposed NonSubsampled pyramid is a 2-D multi-resolution expansion similar to the 1-D NonSubsampled wavelet transform.

\subsection{Nonsubsampled Pyramids}

The multiscale property of the NSCT is obtained from a shift invariant filtering structure that achieves a subband decomposition similar to that of the Laplacian pyramid. This is achieved by using two-channel nonsubsampled 2-D filter banks [4].The nonsubsampled pyramid is completely different from the counterpart of the contourlet transform, the Laplacian pyramid. The building block of the nonsubsampled pyramid is a two-channel nonsubsampled filter bank as shown in Figure 2(a). A nonsubsampled filter bank has no downsampling or upsampling, and hence it is shift-invariant. The perfect reconstruction condition is obtained provided the filters satisfy the Bezout identity: 


$$
H_{O}(z) G_{O}(z)+H_{1}(z) G_{1}(z)=1
$$

This condition is much easier to satisfy than the perfect reconstruction condition for critically sampled filter banks, and thus allows better filters to be designed [5].

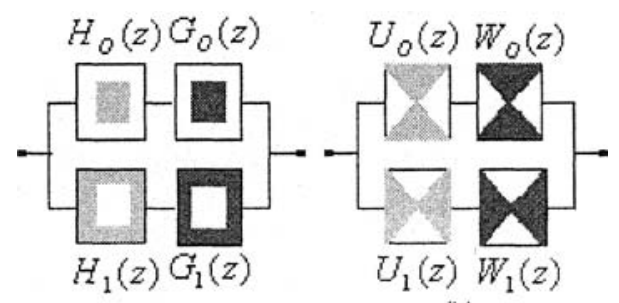

(a)

(b)

Figure 3. Ideal frequency response of the building block of: (a) NonSubsampled Pyramid; (b) NonSubsampled DFB

The ideal frequency response of the building block of the nonsubsampled pyramid is given in Figure 3(a). To achieve the multiscale decomposition, we construct nonsubsampled pyramids by iterated nonsubsampled filter banks. For the next level, we upsample all filters by 2 in both dimensions. Therefore, they also satisfy the perfect reconstruction condition. Note that filtering with the upsampled filter $\mathrm{H}(\mathrm{ZM})$ has the same complexity as filtering with $\mathrm{H}(\mathrm{z})$ using the 'a trous' algorithm. The cascading of the analysis part is shown in Figure 4. These filters achieve multiresolution analysis as shown in Figure 5(a).

\subsection{NonSubsampled Directional Filter Banks}

The nonsubsampled DFB is a shift-invariant version of the critically sampled DFB in the contourlet transform. The building block of a nonsubsampled DFB is also a two-channel nonsubsampled filter bank. However, the ideal frequency response for a nonsubsampled DFB is different, as shown in Figure 3(b). The NSDFB is constructed by eliminating the downsamplers and upsamplers in the DFB. This is done by switching off the downsamplers/upsamplers in each two-channel filter bank in the DFB tree structure and upsampling the filters accordingly.

To obtain finer directional decomposition, we iterate nonsubsampled DFB's. For the next level, we upsample all filters by a quincunx matrix given by

$$
Q=\left(\begin{array}{cc}
1 & 1 \\
1 & -1
\end{array}\right)
$$


The frequency responses of two upsampled filters are given in Figure 6 and the cascading of the analysis part is shown in Figure 7 . Then we obtain a four-direction frequency division as shown in Figure 5(b). The higher level decompositions follow the similar strategy, although they are more complex.

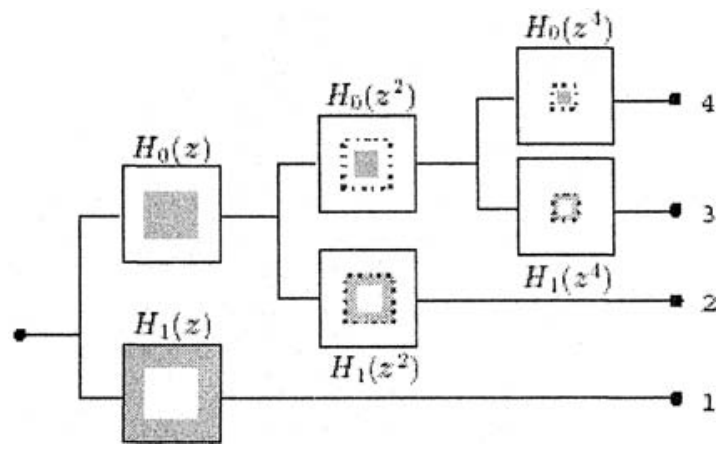

Figure 4. Iteration of two-channel nonsubsampled filter banks in the analysis part of a nonsubsampled pyramid. For upsampled filters, only effective pass bands within dotted boxes are shown.

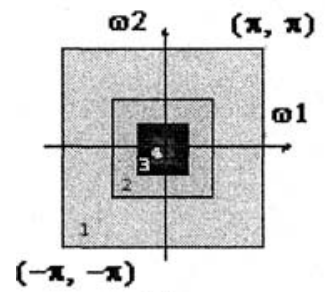

(a)

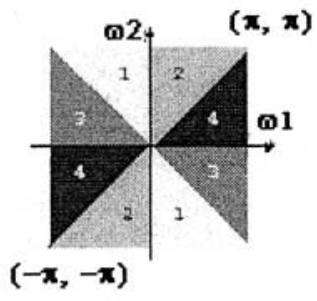

(b)

Figure 5. Frequency divisions of: (a) A NonSubsampled pyramid given in Figure 4. (b) A NonSubsampled DFB given in Figure.7

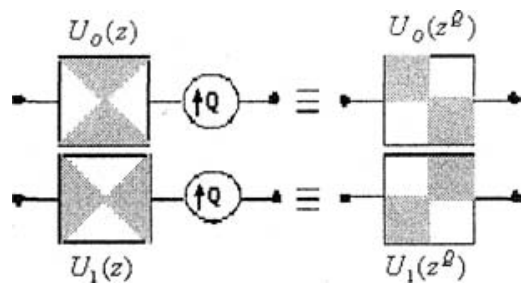

Figure 6. Upsampling filters by a Quincunx matrix $\mathrm{Q}$ 


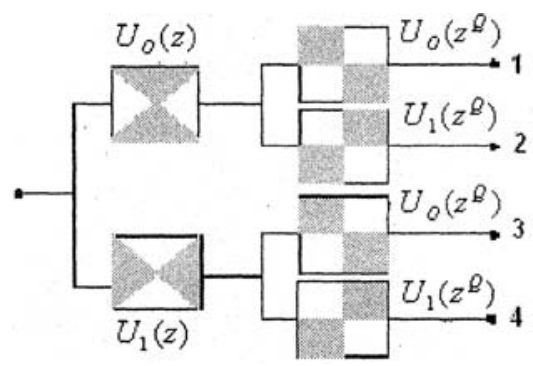

Figure 7. The analysis part of an iterated nonsubsampled directional filter bank.

\subsection{NonSubsampled Contourlet Transform}

The nonsubsampled contourlet transform combines nonsubsampled pyramids and nonsubsampled DFB's as shown in Figure 8. Nonsubsampled pyramids provide multiscale decomposition and nonsubsampled DFB's provide directional decomposition. This scheme can be iterated repeatedly on the low pass subband outputs of nonsubsampled pyramids. First, a nonsubsampled pyramid split the input into a low pass subband and a high pass subband. Then a nonsubsampled DFB decomposes the high pass subband into several directional subbands. The scheme is iterated repeatedly on the low pass subband [5].

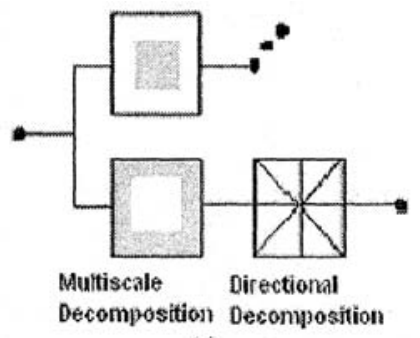

(12)

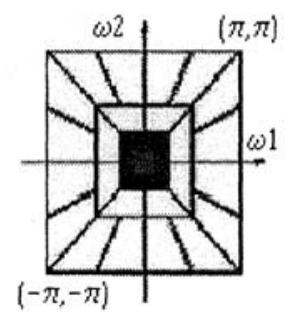

(b)

Figure 8. The NonSubsampled Contourlet Transform: (a) Block diagram. (b) Resulting frequency division, where the number of directions is increased with frequency.

In constructing the nonsubsampled contourlet transform, care must be taken when applying the directional filters to the coarser scales of the pyramid. Due to the tree-structure nature of the NSDFB, the directional response at the lower and upper frequencies suffers from aliasing which can be a problem in the upper stages of the pyramid. Its remedy is to judiciously upsample the NSDFB filters [4]. 


\section{IMAGE ENHANCEMENT ALGORITHM}

The nonsubsampled contourlet transform provides not only multiresolution analysis, but also geometric and directional representation. Since weak edges are geometric structures, while noises are not, we can use this geometric representation to distinguish them. The NSCT is shift-invariant such that each pixel of the transform subbands corresponds to that of the original image in the same location. Therefore, we gather the geometric information pixel by pixel from the NSCT coefficients. It has been observed that there are three classes of pixels: strong edges, weak edges, and noises. First, the strong edges correspond to those pixels with bigvalue coefficients in all subbands. Second, the weak edges correspond to those pixels with big-value coefficients in some directional subbands but small-value coefficients in other directional subbands within the same scale. Finally, the noises correspond to those pixels with small-value coefficients in all subbands. Based on this observation, pixels can be classified into three categories by analyzing the distribution of their coefficients in different subbands. One simple way is to compute the mean (denoted by mean) and the maximum (denoted by max) magnitude of the coefficients for each pixel, and then classify it by

$$
\left\{\begin{array}{l}
\text { StrongEdge if mean } \geq c \sigma \\
\text { WeakEdge if mean }<c \sigma, \max \geq c \sigma \\
\text { Noise } \quad \text { if mean }<c \sigma, \max <c \sigma
\end{array}\right.
$$

where $\mathrm{c}$ is a parameter ranging from 1 to 5 , and is the noise standard deviation of the subbands at a specific level. We first estimate the noise variance of the input image with the robust median operator and then compute the noise variance of each subband. The goal of image enhancement is to amplify weak edges and to suppress noises. To this end, we modify the NSCT coefficients according to the category of each pixel by a nonlinear mapping function.

$$
y(x)= \begin{cases}x & \text { StrongEdgePixels } \\ \max \left(\left(\frac{c \sigma}{|x|}\right)^{\mathrm{p}}, 1\right) x, & \text { WeakEdgePixels } \\ 0 & \text { NoisePixels }\end{cases}
$$

where the input $x$ is the original coefficient, and $0<p<1$ is the amplifying ratio. This function keeps the coefficients of strong edges, amplifies the coefficients of weak edges, and zeros the coefficients of noises. We summarize our enhancement method using the NSCT in the following algorithm:

1. Compute the NSCT of the input image for $N$ levels. 
2. For each level DFB,

a) Estimate the noise variance,

b) Compute the threshold and the amplifying ratio.

c) At each pixel, compute the mean and the maximum magnitude of all directional subbands at this level, and classify it by (3) into strong edges, weak edges, or noises.

d) For each directional subband, use the nonlinear mapping function given in (4) to modify the NSCT coefficients according to the classification.

3. Reconstruct the enhanced image from the modified NSCT coefficients.

4. Calculate the Detail and Background Variance.

\section{EXPERIMENTAL RESULTS}
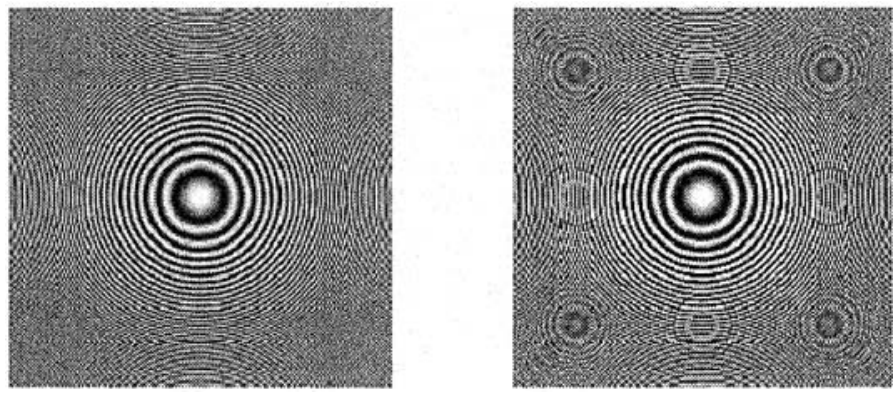

Figure 9. (a) Original Zoneplate image. (b) Enhanced by the NonSubsampled Contourlet Transform
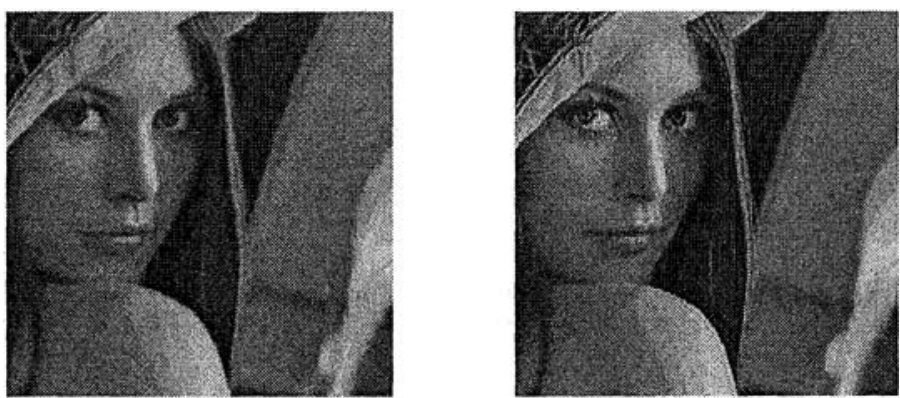

Figure 10. (a) Original Lena image. (b) Enhanced by the NonSubsampled Contourlet Transform 

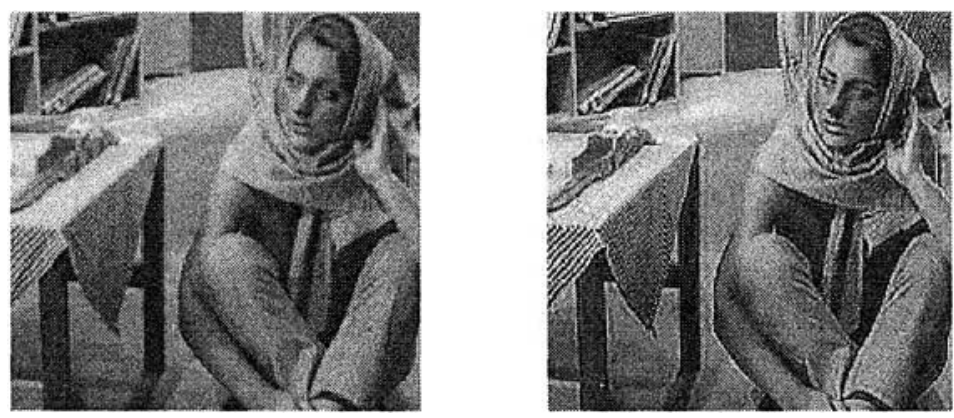

Figure 11. (a) Original Lena image (b) Enhanced by the NonSubsampled Contourlet Transform
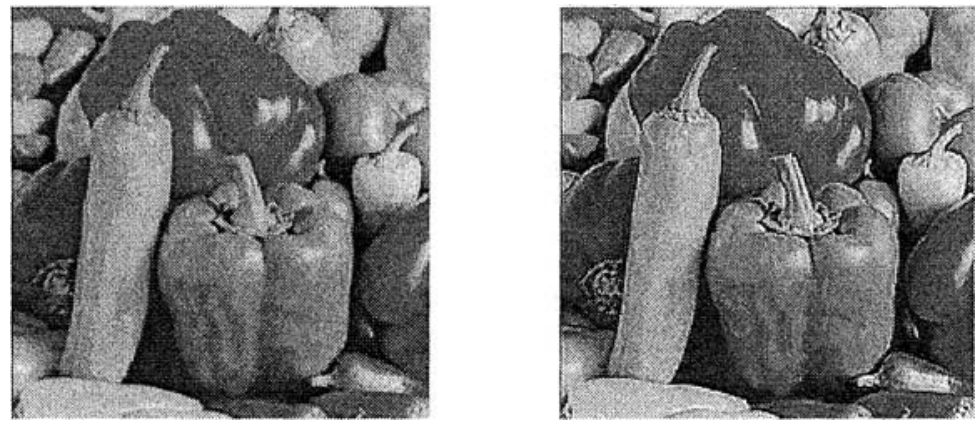

Figure 12. (a) Original Peppers image. (b) Enhanced by the NonSubsampled Contourlet Transform.

Table 1. Detail and Background Variance of different Images

\begin{tabular}{rrrcc}
\hline \multicolumn{1}{c}{ Image } & \multicolumn{2}{c}{ Original Image } & \multicolumn{2}{c}{ Reconstructed Image } \\
\hline & Detail & Background & Detail Variance & Background \\
Variance (DV) & Variance (BV) & (DV) & Variance (BV) \\
Zone plate & 8186.665 & 114.383 & 69244.110 & 816.018 \\
Lena & 692.266 & 25.150 & 1061.337 & 38.227 \\
Barbara & 805.487 & 55.447 & 4379.570 & 113.118 \\
Peppers & 1142.621 & 32.987 & 2842.605 & 78.889 \\
\hline
\end{tabular}

To evaluate the enhancement performance objectively, the detailed variance (DV) and background variance (BV) is calculated. The DV and BV values represent the variance of foreground and background pixels, respectively. Detail and background variance is calculated around every pixel by taking the variance of 
image intensities and classifying the pixel into foreground or background based on a threshold. The average of variance of foreground pixels gives Detail Variance (DV) and the average of variance of background pixels gives Background Variance (BV). A good enhancement method should increase the DV of the original image but not the BV. It has been observed that proposed algorithm offers better results in enhancing the weak edges in the textures.

\section{CONCLUSION}

The proposed nonsubsampled Contourlet transform is constructed by iterated nonsubsampled filter banks. This transform provides shift-invariant directional multiresolution image representation. This new algorithm for image enhancement using the nonsubsampled Contourlet transform will show that better enhancement results can be obtained than the previous enhancement techniques.

\section{REFERENCES}

1. Rumin Eslumi und Huyder Rudhu, "Wavelet based Countourlet Transform and its application to Image Coding", ECE Department, Michigan State University, East Lansing, MI 48824, USA.

2. Minh N. Do and Martin Vetterli, "The Countoulet Transform: An Efficient Directional Multiresolution Image Representaion", IEEE, Trans. Image Proc., 2005.

3. Panchamkumar D SHUKLA, Department of Electronic and Electrical Engineering, "Complex Wavelet Transform And Their Applications", University of Strathclyde, Glasgow G1 1XW, Scotland, United Kingdom.

4. L. Cunha, J. Zhou, and M. N. Do, "The nonsubsampled contourlet transform: theory, design and applications," IEEE Trans. Image Proc., submitted, 2005.

5. L. Cunha, J. Zhou, and M. N. Do, "Nonsubsampled Contourlet Transform: Construction and Application in Enhancement," IEEE Trans. Image Proc, submitted, 2005. 